\title{
Cable Failure Operation Strategy for a Rehabilitation Cable-Driven Robot ${ }^{\dagger}$
}

\author{
Giovanni Boschetti ${ }^{1, *}$, Giuseppe Carbone ${ }^{2}$ and Chiara Passarini ${ }^{1}$ \\ 1 Department of Management and Engineering-DTG, University of Padova, 36100 Vicenza, Italy; \\ chiara.passarini@phd.unipd.it \\ 2 Department of Mechanical, Energy and Management Engineering, University of Calabria, 87036 Rende, \\ Italy; giuseppe.carbone@unical.it \\ * Correspondence: giovanni.boschetti@unipd.it; Tel.: +39-0444-99-8748 \\ + This paper is an extended version our paper published in Boschetti, G.; Carbone, G.; Passarini, C. A Fail-Safe \\ Operation Strategy for LAWEX (LARM Wire driven EXercising device). In the Proceedings of the 4th \\ IFToMM Symposium on Mechanism Design for Robotics, Udine, Italy, 11-13 August 2018.
}

Received: 24 January 2019; Accepted: 2 March 2019; Published: 6 March 2019

\begin{abstract}
Cable-Driven Parallel Robots (CDPR) have attracted significant research interest for applications ranging from cable-suspended camera applications to rehabilitation and home assistance devices. Most of the intended applications of CDPR involve direct interaction with humans where safety is a key issue. Accordingly, this paper addresses the safety of CDPRs in proposing a strategy to minimize the consequences of cable failures. The proposed strategy consists of detecting a cable failure and avoiding any consequent motion of the end-effector. This is obtained by generating a wrench that is opposite to the direction of the ongoing motion so that the end-effector can reach a safe position. A general formulation is outlined as well as a specific case study referring to the LAWEX (LARM Wire-driven EXercising device), which has been designed within the AGEWELL project for limb rehabilitation. Real-time calculation is carried out for identifying feasible cable tensions, which generate a motion that provides the desired braking force. Simulations are carried out to prove the feasibility and effectiveness of the strategy outlined here in cases of cable failure.
\end{abstract}

Keywords: cable-driven parallel robots; fail-safe operation; exercising device

\section{Introduction}

Cable-driven parallel robots (referred to as CDPR in this paper) are parallel robots where the end-effector is linked to the base platform by replacing traditional rigid links with cables. This provides significant advantages, for example, in speed, acceleration, and workspace as compared with traditional robots. The abovementioned features make CDPRs an attractive alternative to traditional parallel robots. Cable-suspended cameras are widely known and even commercially implemented as applications of CDPR [1]. Moreover, many prototypes have been proposed in fields such as rehabilitation and home care [2]. Besides their numerous advantages, CDPRs introduce an additional constraint to motion planning and control, since cables can only exert positive tension forces on the end-effector. This characteristic makes trajectory planning and control tasks very complex. Moreover, CDPRs are more difficult to brake as compared with traditional robots with rigid links [3]. In fact, in traditional robots a hard stop of motors quickly leads to a full stop of the whole robot, while this cannot be applied to CDPRs since a hard stop of motors in CDPRs cannot prevent further motion of the flexible cables that allow motion in the robot end-effector. This makes it very difficult to create an emergency stop for CDPRs, while this feature would be very important from a safety point of view.

The failure of cables has hardly been addressed in the literature on cable-driven robots. An example can be found in [4], where the authors investigated the possibility of removing a cable 
while maintaining a static equilibrium pose. In particular, the authors of [4] proposed a method to identify the cables that are critical for ensuring the feasibility of a specific configuration. Then, the method finds out any unnecessary cable. In [5] and further in [6], Notash presented a possible classification of the feasible failure modes and introduced three methods to recover or compensate for a lost wrench of a redundant cable robot in static equilibrium conditions. In [7] the authors suggest using an optimal design procedure for minimizing the differences in cable tensions. This approach can also be applied in the case of a cable failure.

A completely different approach is presented in [8], where the authors propose a first method to control a moving end-effector after a cable failure. Such a strategy consists of generating a safe motion to a predetermined pose, while keeping the end-effector inside the workspace. The planning of such motion is based on pre-computed force limits and trajectories. A different approach was presented in [9], where the end-effector reaches the safe position by following a planned oscillatory trajectory. Such a trajectory still allows us to achieve a positive and bounded tension in each cable.

This paper improves on the approach proposed in [8] by proposing an efficient way to calculate and achieve a straight-line trajectory after failure, specifically for the case of the LAWEX robot $[10,11]$. This represents a first attempt to apply the Wrench Exertion Capability [12,13] as a tool to manage safety in cable-driven robots. It is important to note that this work is aiming at a preliminary feasibility study. Accordingly, only one type of cable failure has been considered, while other more complex cases will be considered as future work, with specific experimental validations with end users. The current work will preliminarily focus on the case of a single cable failure where the robot shape is preserved. In this case, even if the workspace keeps a shape similar to the original one, the configuration of the robot changes from an overconstrained to a suspended configuration. This change is very important, since a suspended CDPR must rely on gravity to maintain positive tension in all cables [14]. Moreover, when the robot configuration changes due to failure, its performance in terms of force exertion capability decreases unpredictably and the planned motion can become unfeasible. Hence, it is important to have a control tool that can be used in real time to estimate the feasible wrench and identify which cable tensions are needed to stop the end-effector in case of cable failure. Future works will investigate the possibility of applying the Wrench Exertion Capability to more complex CDPRs, as in [15].

The paper is organized as follows: Section 2 provides a description of the design and the key operation characteristics of LAWEX; Section 3 describes the proposed motion strategy in case of a cable failure; Section 4 provides a description of the main characteristics of LAWEX in terms of workspace and forces; Section 5 gives simulation results for the proposed motion strategy in case of one cable failure; finally, Section 6 gives the conclusions.

\section{Characteristics of LAWEX Robot}

In the last decade, a research team led by the second author at LARM has been investigating several cable-driven parallel robot architectures within the European co-funded project AGEWELL, currently underway at Technical University of Cluj-Napoca, Romania. Specifically, this project aims to investigate novel devices and design solutions for limb rehabilitation tasks, as reported for example in $[10,11]$. Within this frame a novel cable-driven design solution has been proposed and built as shown in Figure 1. The name of the prototype in Figure 1 is LAWEX (LARM Wire-Driven EXercising device). Its design has been based on preliminary studies that have been carried out on human patients to determine the desired limb motions, such as reported in [16-19]. The main innovative aspect of LAWEX is its open architecture, which can allow easy accessibility by users during treatment, even if they are sitting in a wheelchair. Aluminum profiles are used for a lightweight and stiff design, while the robot structure can be easily assembled, stored, and moved so that it can be suitable even for home use. 


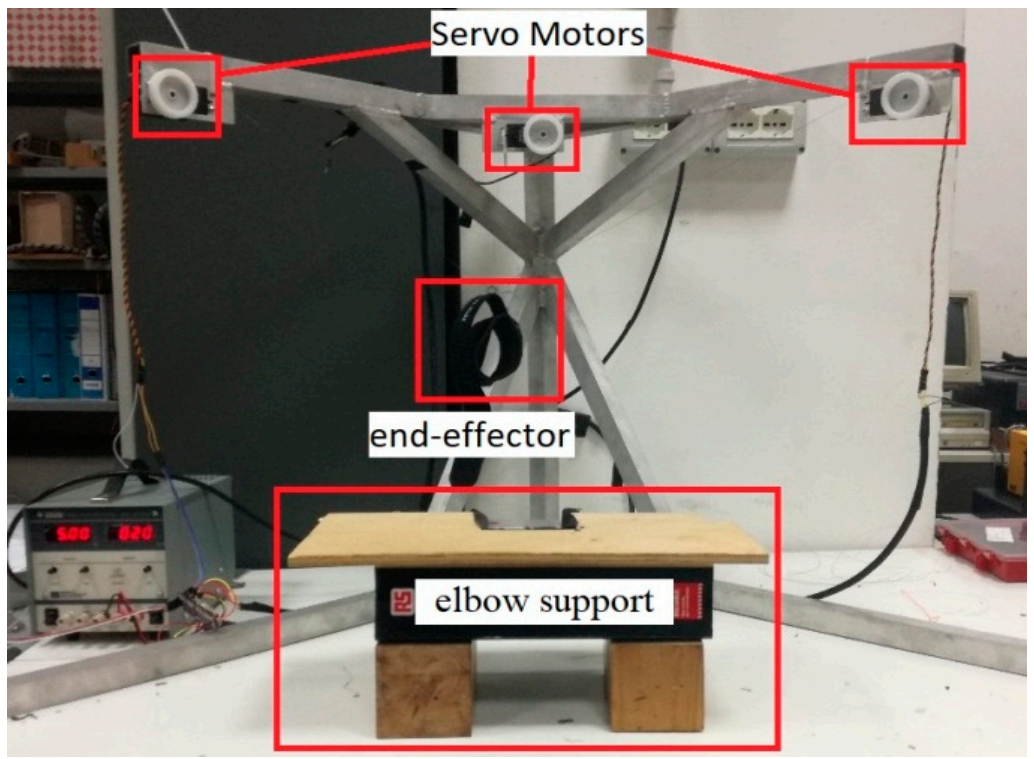

Figure 1. A built prototype of the LAWEX robot. (The main components are highlighted with red boxes.)

The LAWEX robot includes four servo motors and four cables; the cables are connected to the end-effector through a wristband as shown in Figure 1. Moreover, Figure 2 outlines an operation scheme of LAWEX. Namely, the human arm is attached to an interface/end-effector. This interface/end-effector is attached to a rigid frame by means of four cables. Each cable is connected to a servomotor that can change the cable length by using a winch. One of the servomotors is below the platform; the other three are attached to the upper part of the rigid frame. Cables are connected to the end-effector on the arm to be trained using a wristband. Further details on the end-effector are reported in [17]. This setup allows several different motions that are suitable for limb exercises such as for rehabilitation purposes.

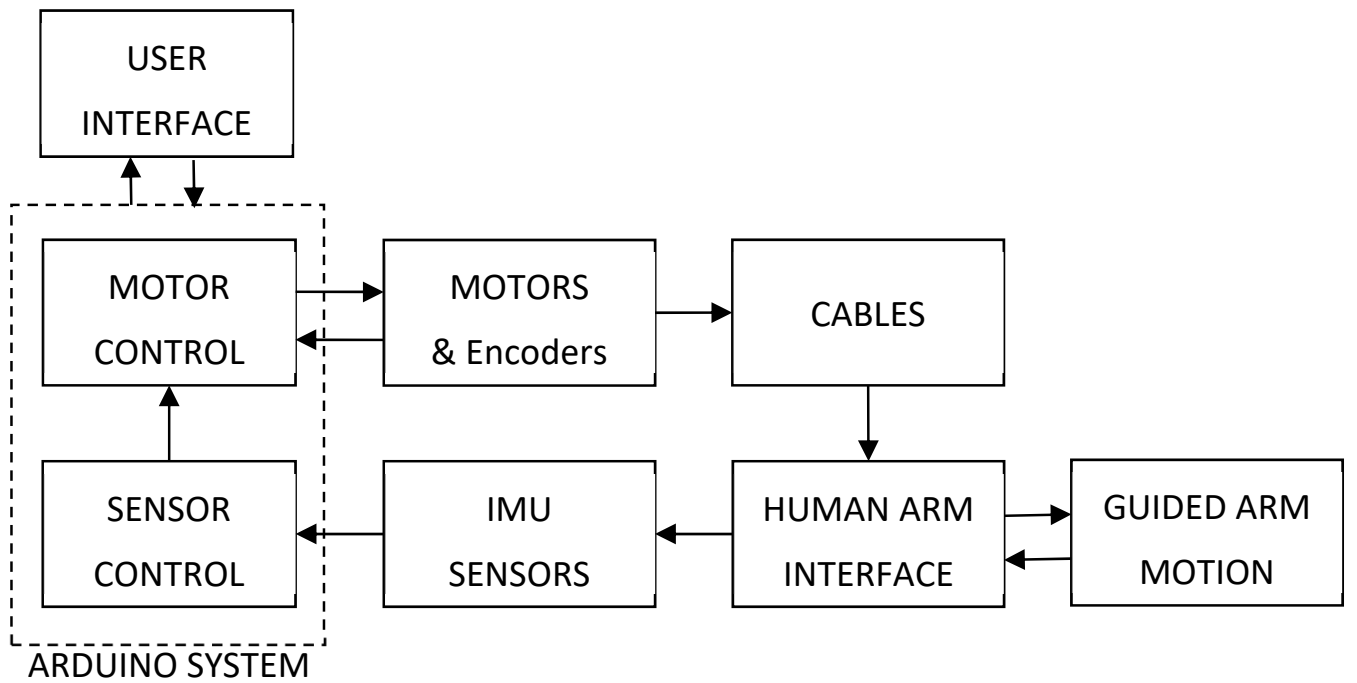

Figure 2. A scheme of the operation architecture of LAWEX.

A successful rehabilitation procedure relies on several key aspects. In particular, it is necessary to define and complete a reliable and safe motion based on a proper training protocol. Accordingly, several experiments have been carried out to set up the proper motion parameters such as the elbow support position and arm motion ranges. Some tested setup conditions are shown in Figure 1, where an elbow support keeps the elbow position fixed relative to LAWEX. This limits the arm motion to elbow flexion-extension. 


\subsection{Kinematics}

The LAWEX frame structure has one servomotor in its bottom edge, where the fixed reference frame $X Y Z$ is also located. Three other servomotors are attached on the upper part of the robot frame structure, one at the top edge and the other two at the top extremities of the structure, as also shown in Figure 1. A kinematic scheme of LAWEX is shown in Figure 3, which gives the location of cables. In this figure the starting points of cables are indicated by using the position vector $A_{i}$ $(i=1,2,3,4)$, where each starting point refers to the attachment of a cable to its pulley that is attached to the corresponding servomotor. Accordingly, one can write:

$$
A_{1}=\left(-a_{1} ;-a_{2} ; h\right), A_{2}=(0 ; 0 ; h), A_{3}=\left(a_{1} ;-a_{2} ; h\right), A_{4}=(0 ; 0 ; 0) .
$$

The pose of the end-effector EE in Figure 3 is defined by the cable connection points $B_{i r}$, $(i=1,2,3,4)$, whose positions can be defined in reference to point $\mathrm{H}$ in the center of $\mathrm{EE}$ (this can also be defined as the mobile platform), such as:

$$
B_{1 r}=\left(-b_{1} ;-b_{2} ; 0\right), B_{2 r}=\left(0 ; b_{3} ; 0\right), B_{3 r}=\left(b_{1} ;-b_{2} ; 0\right), B_{4 r}=(0 ; 0 ; 0)
$$

If one assumes a fixed orientation of the moving platform during the robot operation, the absolute position of point $H(x ; y ; z)$ can be easily obtained by means of a loop-closure equation for the $i$-th kinematic chain such as:

$$
H+B_{\text {ir }}=A_{i}+E_{i}
$$

where $E_{i}$ is the vector representing the $i$-th cable of the robot. Therefore, considering Equation (3), one can write:

$$
E_{i}=H+B_{i r}-A_{i}
$$

In this way it is possible to solve the inverse kinematics problem of the cable robot by computing the vector modules on both sides as:

$$
\left\|E_{i}\right\|=\left\|H+B_{i r}-A_{i}\right\|
$$

The relationships between the cable lengths and the position of the point $\mathrm{H}$ on the EE can be expressed as:

$$
\begin{gathered}
l_{1}=\sqrt{\left(x+a_{1}+b_{1}\right)^{2}+\left(y+a_{2}+b_{2}\right)^{2}+(z-h)^{2}} \\
l_{2}=\sqrt{x^{2}+\left(y-b_{3}\right)^{2}+(z-h)^{2}} \\
l_{3}=\sqrt{\left(x-a_{1}-b_{1}\right)^{2}+\left(y+a_{2}+b_{2}\right)^{2}+(z-h)^{2}} \\
l_{4}=\sqrt{x^{2}+y^{2}+z^{2}}
\end{gathered}
$$




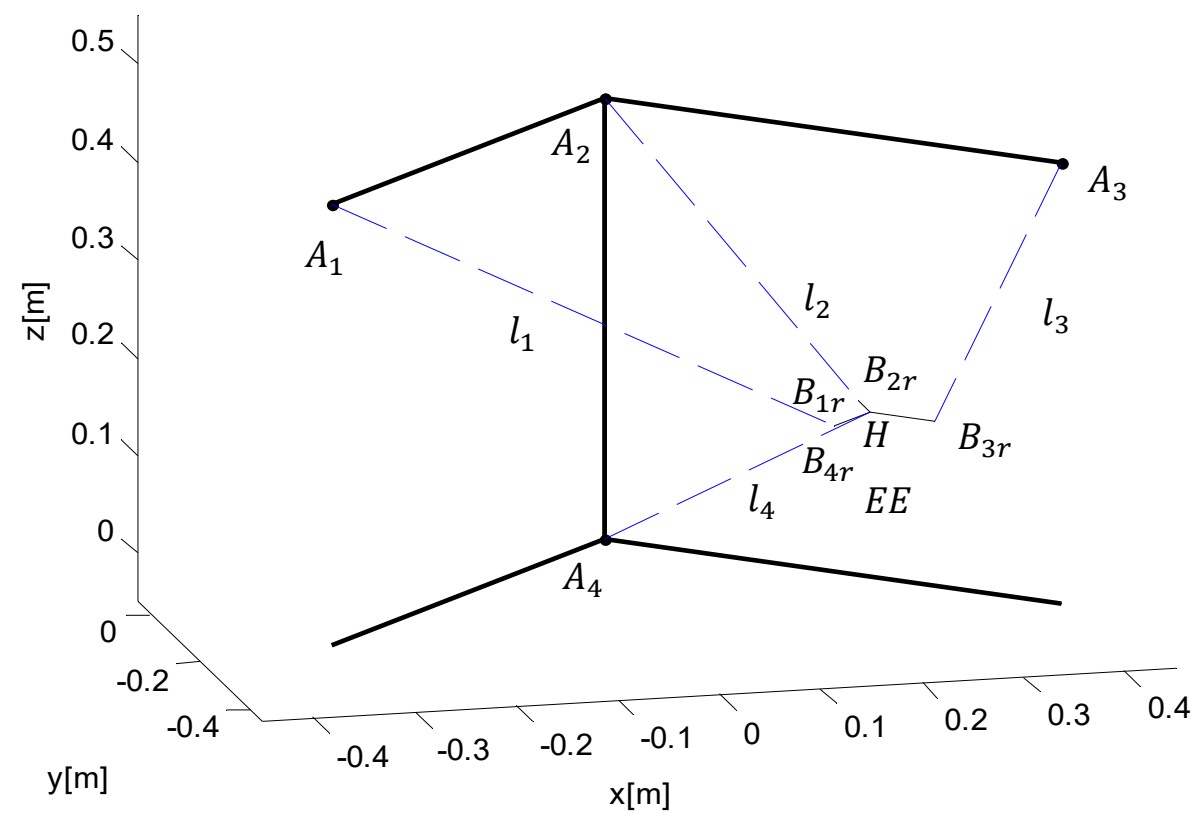

Figure 3. The kinematic scheme of the LAWEX architecture.

\subsection{Dynamics}

If one neglects the elasticity of the cables, the length of each cable can be considered as directly proportional to the angular displacement $\vartheta_{i}$ of the servomotors according to the relationship

$$
l_{\mathrm{i}}=l_{0}-\vartheta r_{0}-\frac{\pi r_{1}}{2}-d_{0}
$$

where $l_{0}$ is the total length of the cable, $r_{o}$ is the driving pulley radio, $r_{1}$ is the secondary pulley radio, and $d_{0}$ is the distance between the centers of the pulleys. By isolating the term $\vartheta_{i}$, it is possible to obtain the actuation vector of the manipulator for computing the corresponding motion angle, which allows the actuators to reach the desired position.

The dynamic model of this manipulator can be described by

$$
\boldsymbol{w}=\left[\begin{array}{c}
f_{x} \\
f_{y} \\
f_{z}
\end{array}\right]=\left[\begin{array}{lllll}
\boldsymbol{u}_{1} & \boldsymbol{u}_{2} & \boldsymbol{u}_{3} & \boldsymbol{u}_{4} & \boldsymbol{u}_{g}
\end{array}\right]\left[\begin{array}{c}
\tau_{1} \\
\tau_{2} \\
\tau_{3} \\
\tau_{4} \\
m g
\end{array}\right]=S \boldsymbol{\tau}
$$

where $w$ is the wrench vector that is represented by the three exerted forces $f_{i} ; S$ is the structure matrix that is made by the normal vectors $\boldsymbol{u}_{i}$. Each vector $\boldsymbol{u}_{i}$ represents the direction that is connecting the end-effector with the $i$-th pulley; $\tau$ is the vector that contains the cable tensions $\tau_{i}$. The abovementioned mg represents the gravity force, which can be considered as equivalent to a vector with constant direction and constant modulus.

\subsection{Programming the LAWEX Operation}

The programming of LAWEX has been developed according to Figure 4 . In particular, Figure $4 \mathrm{~b}$ shows a specifically developed user-friendly interface of LAWEX that runs in Java and can also be operated with Android smartphones. The user interface includes a button to perform the path planning. This is obtained by using the inverse kinematics of LAWEX, as discussed in the previous section and in [13]. The obtained results of the inverse kinematics are the cable lengths versus time, with which we are able to generate the desired path of the end-effector. The computed cable lengths are then converted 
into the angular positions of the servomotors. This is done by considering that the rotation of each servomotor winds or unwinds its cable about its own winch. Each winch consists of a cylindrical drum of known diameter. Accordingly, each servomotor rotation generates a known increase or decrease of the cable length. The computed vector of angular positions versus time is sent to an Arduino board, which drives all four servomotors.

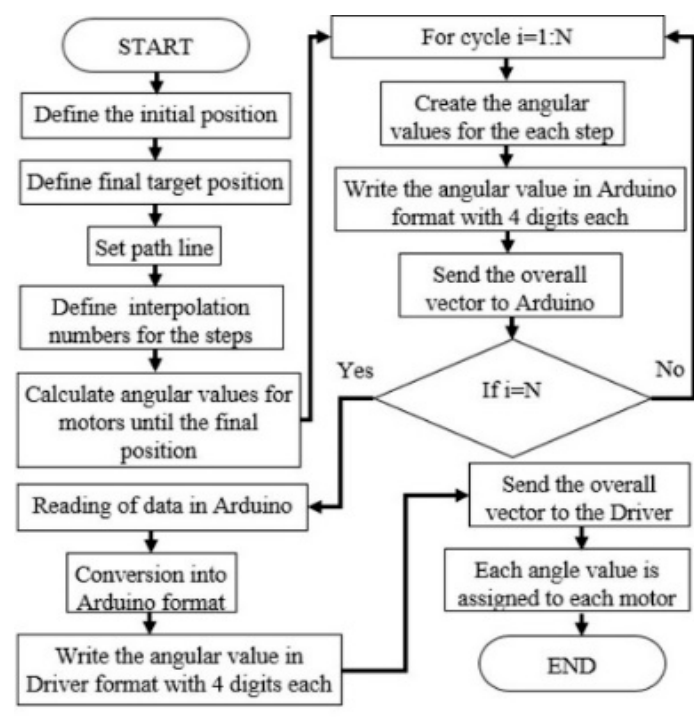

(a)

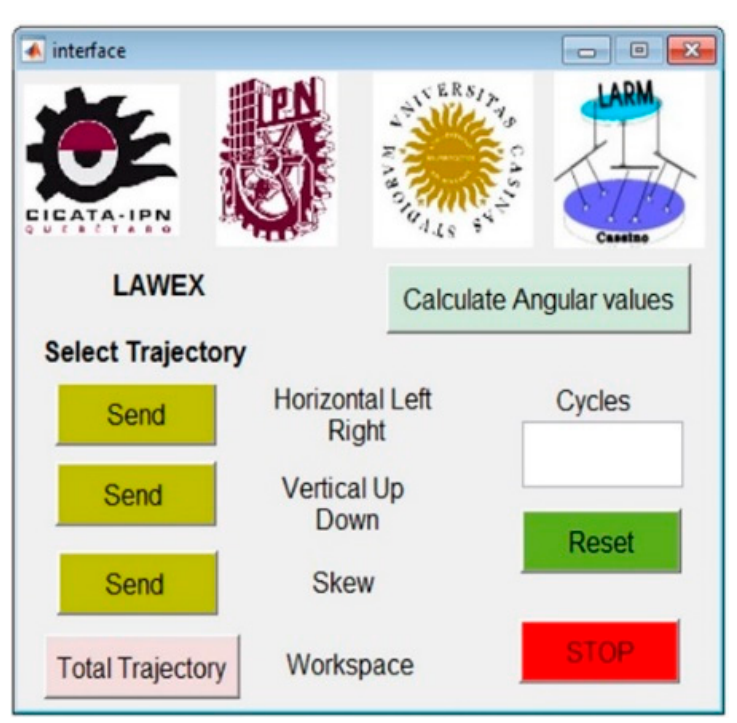

(b)

Figure 4. The developed user interface of LAWEX: (a) flowchart of the software architecture; (b) the main interface screen.

The path planning can be generated in real time for short motions or off-line for predefined motion paths such as horizontal motions from left to right and vice versa, vertical motions up and down and vice versa, or a skew (diagonal) motion combining the previous two pre-defined paths. The latter achieves up-down motion followed by left-right motion. For each of these predefined paths it is possible to set a desired number of cycles $N$ so that the path is cyclically repeated $N$ times. The button "calculate angular values" starts the calculations for path planning and sends the output vector of angular positions to the Arduino board. One can set other trajectories through the "total trajectory" button by setting up a list of the point coordinates one wishes to reach versus time.

If the system has a failure, one can push the reset button and the end-effector returns to its initial configuration, which means it is at top up configuration and centered. One can also push the stop button in an emergency.

\section{Motion Strategy after Failure}

The main goal of the proposed strategy is planning and achieving a motion for reaching a safe position after a cable failure. In this application, such a strategy takes into account the Wrench Exertion Capability (WEC) of the rehabilitation robot and allows the motors to exert a braking force in the opposite direction of motion. To make sure that the strategy works, the end-effector should also be inside the feasible workspace after the cable failure. In the proposed case, this condition is certainly respected when cable 4 breaks: indeed, when another cable breaks, the workspace can degenerate into a bidimensional shape and the motion of the end-effector cannot be easily controlled. It is worth noting that, during a therapy rehabilitation task, cable 4 can reach tensions higher than the other cables. Therefore, it might be advisable to increase the number of cables to distribute the corresponding load on more cables in a redundant setup.

The force that can be exerted in a specific direction, before and after the cable failure, can be computed by means of a performance index called Wrench Exertion Capability (WEC). As mentioned 
above, this index computes the maximum force (or torque) that can be exerted in a given direction while keeping all the other components null. This index can be used to compute the maximum exertable braking force in the direction of motion, aimed at stopping the end-effector. It is important to note that a specific reference frame should be defined in order to compute the WEC index. Such a reference frame should have an axis that lies along the direction of motion d. Accordingly, the braking force is computed by searching for the cable tensions that guarantee the maximum braking force along the direction of motion $\mathrm{d}$ by referring to the axis of the abovementioned reference frame. The following linear programming problem allows for achieving the proper cable tension:

$$
\begin{gathered}
\text { minimize }:\left(f_{d}\right)=\boldsymbol{s}_{d} \boldsymbol{\tau} \\
\text { s.t. : }\left\{\begin{array}{c}
\boldsymbol{S}_{o} \boldsymbol{\tau}=\mathbf{0} \\
\boldsymbol{\tau}_{\text {min }} \preccurlyeq \boldsymbol{\tau} \preccurlyeq \boldsymbol{\tau}_{\text {max }} \\
\tau_{f}=0
\end{array} \forall \tau \neq \tau_{f},\right.
\end{gathered}
$$

where

- $s_{d}$ is defined as the row of the Structure Matrix $S_{r}$ that refers to the direction of motion. Let us define $R$ as the rotation matrix that identifies the novel reference frame $S_{r}$ by means of the following relation: $S_{r}=R S$;

- $S_{o}$ is the matrix obtained by $S_{r}$ by removing the row $s_{d}$;

- $\tau_{\min }$ and $\boldsymbol{\tau}_{\max }$ are the vectors that define the range of the proper cable tensions;

- $\tau_{f}$ identifies the broken cable, whose tension is set to a null value.

From a practical point of view, following a cable failure, a wise choice can be to reduce the value of the maximum allowed cable tension in order to reduce the risk of another cable failure. Therefore, in this application we assume the maximum allowed cable tension after failure to be reduced to $70 \%$ of the maximum allowed cable tension before failure so that $\tau_{\max E}=0.7 \times \tau_{\max }$.

An important issue that is related to optimization problems is that they are iterative and time-consuming. For this reason, their application in a real-time environment is discouraged and sometimes not applicable. This aspect is thus carefully considered to keep the computational costs within a feasible range for real-time implementation. Accordingly, the proposed case study considers a computationally efficient approach for computing the WEC index and the maximum allowed braking force as soon as a cable failure is detected. The chosen method for computing the WEC index has already been proposed in [13]. It is based on a geometric representation of the forces that can be exerted. The main idea behind the algorithm is to consider the possible exertable wrenches as a polytope in the $\mathrm{n}$-dimensional space (where $\mathrm{n}$ is the number of degrees of freedom). The $\mathrm{n}$-dimensional space is represented so that the $x$-axis is oriented with the direction of interest $\mathrm{d}$. The intersections between the $x$-axis and the skull of the wrench polytope represent the maximum and minimum exertable forces, respectively, in the direction of interest. The proposed algorithm also allows the corresponding tension configuration able to exert such forces. The corresponding tension vector is in the skull of the tension polytope in the $\mathrm{m}$-dimensional case (where $\mathrm{m}$ is the number of cables). This algorithm allows for cutting $80 \%$ of the computational time compared with classic optimization-based algorithms.

\section{Performance Analysis}

This section reports a preliminary analysis of the performance (in terms of workspace and exertable force) of the cable robot before and after failure. The structure of the robot is described in Section 2. Considering the sizes of LAWEX, parameters $a_{1}, a_{2}$ are both set to $0.36 \mathrm{~m}$; $h$ is $0.46 \mathrm{~m}$, while $b_{i}$ can be neglected. For the performance analysis the origin of the fixed reference frame is attached to point $A_{2}$. Figure 5 represents the static equilibrium workspace before and after failure. Figure 5 is obtained by setting a minimum tension of $0.5 \mathrm{~N}$ and a maximum acceptable tension of $10 \mathrm{~N}$ as referring to the twisted iron cables of LAWEX. The mass of the end-effector is equal to $5 \mathrm{~N}$ (robot 
unload). On the other hand, the force exertion capability has been investigated by considering the directions of motion that the robot is supposed to have, i.e., left-right and up-down. The left-right motion in the considered reference frame results in a straight-line motion in the direction $45^{\circ}$ in the plane $x-y$. The cyan rectangle in Figure 5 represents the plane for which the performance regarding the right-left motion has been investigated. Simulations suggest that the best performance for this motion can be reached when $\mathrm{z}=-\mathrm{h} / 2$. Figure 6 shows the computed maximum exertable force in the right direction $(a-c)$ and in the left direction $(b-d)$. This has been computed before $(a-b)$ and after $(c-d)$ the failure of the cable with a color indicating the force magnitude. The color scale in $[\mathrm{N}]$ is reported on the right side of Figure 6 . The shape of the polytope changes according to the position of the end-effector, since it is strictly related to the structure matrix $S$. It is important to note that only the tension polytope after failure is shown here, since it has a three-dimensional shape. Instead, the tension polytope before failure is a four-dimensional polytope that is not representable in a 3D plot.

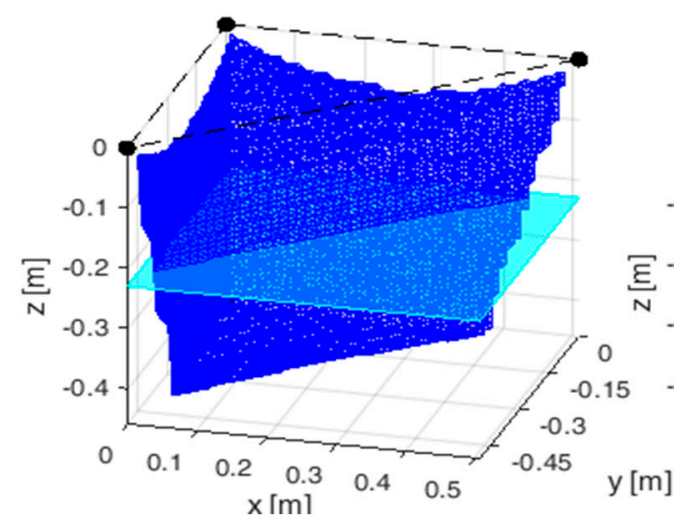

(a)

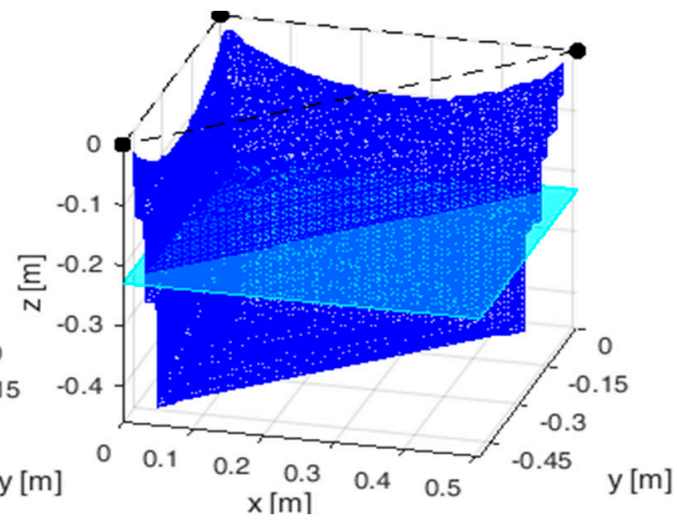

(b)

Figure 5. Workspace of LAWEX before (a) and after (b) failure.
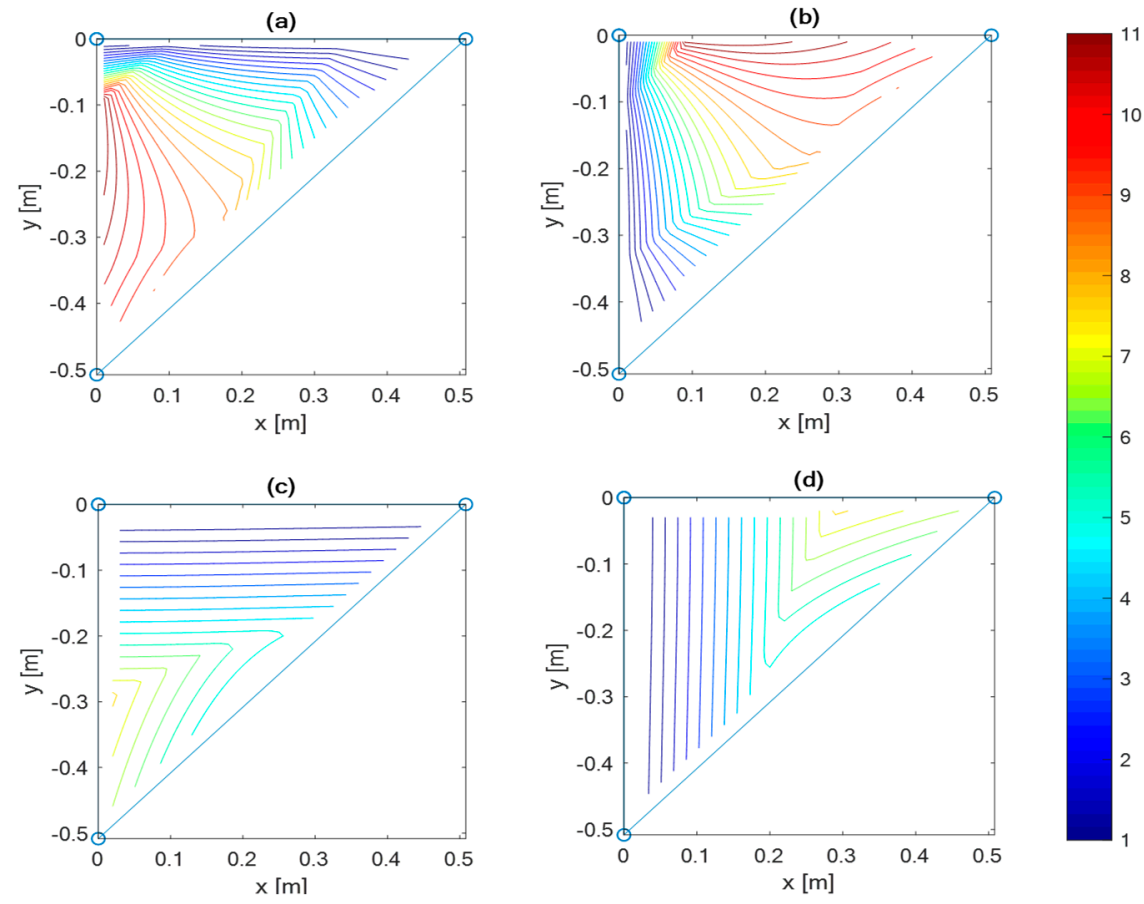

Figure 6. Computed maximum exertable force in the right direction $(\mathbf{a}-\mathbf{c})$ and in the left direction (b-d) before $(\mathbf{a}, \mathbf{b})$ and after $(\mathbf{c}, \mathbf{d})$ the failure of the cable. 


\section{Simulations}

This section addresses the simulation of the behavior of LAWEX within its operation workspace. Discussion is also provided to clarify the main differences between the proposed approach and other previous approaches that are available in the literature, such as those reported in $[5,6]$.

\subsection{Simulation with Previous Approaches}

Previous approaches are conceptually totally different from the proposed one as they aim to compensate for the lost wrench due to a cable failure by mostly considering the static equilibrium conditions.

A numerical simulation is reported to clarify the main features of the approaches in literature by referring to the case study of LAWEX. Namely, one can consider the LAWEX robot with the end-effector lying within its static feasible workspace. Without lack of generality, a feasible generic point $P$ has been chosen with coordinates $P(0.05,-0.2,0.23)$. In this position the static equilibrium is guaranteed by the vector of cable tensions $\tau$ that allows one to achieve a null wrench $w$ in Equation (11) so that:

$$
\boldsymbol{w}=\left[\begin{array}{lllll}
\boldsymbol{u}_{1} & \boldsymbol{u}_{2} & \boldsymbol{u}_{3} & \boldsymbol{u}_{4} & \boldsymbol{u}_{g}
\end{array}\right]\left[\begin{array}{c}
\tau_{1} \\
\tau_{2} \\
\tau_{3} \\
\tau_{4} \\
m g
\end{array}\right]=S \boldsymbol{\tau}=\left[\begin{array}{l}
0 \\
0 \\
0
\end{array}\right]
$$

Since the robot is redundant, there are infinite tension vectors that satisfy Equation (14). In this case the solution with the minimum tension is chosen. Indeed, in this case one of the cables exerts the minimum allowed tension, $0.5 \mathrm{~N}$, so that the vector of cable tensions $\tau$ is: $\tau=\{2.62,2.94,3.67,0.5, m g\}$.

If one considers the failure of one cable, its tension becomes zero. In case of failure of the fourth cable, its tension became null $\left(\tau_{4}=0\right)$. The existing methods in the literature, such as $[5,6]$, aim at achieving a new set of cable tensions that satisfies Equation (4) under the condition that one cable is no longer able to exert any force. Accordingly, one can compute the wrench vector $w$ as follows:

$$
\boldsymbol{w}=\left[\begin{array}{lllll}
\boldsymbol{u}_{1} & \boldsymbol{u}_{2} & \boldsymbol{u}_{3} & \boldsymbol{u}_{4} & \boldsymbol{u}_{g}
\end{array}\right]\left[\begin{array}{c}
\tau_{1} \\
\tau_{2} \\
\tau_{3} \\
0 \\
m g
\end{array}\right]=S \boldsymbol{\tau}=\left[\begin{array}{l}
0 \\
0 \\
0
\end{array}\right] .
$$

In this case, the robot is no longer redundant, since one cable is considered broken. Accordingly, there is only one feasible tension vector that satisfies Equation (15) and vector $\tau$ can be computed as $\tau=\{2.29,2.99,3.19,0, m g\}$. This new tension vector allows for maintaining the static equilibrium of the end-effector after a cable failure.

\subsection{Simulation of the Proposed Motion Strategy}

The proposed approach differs totally from other existing methods, since it deals with a cable failure by planning a motion strategy under dynamic conditions, instead of addressing the static case as in existing methods. A numerical simulation is reported here to clarify the main features of the proposed novel approach by referring to the case study of LAWEX.

An algorithm has been written in Matlab-Simulink in order to prove its effectiveness. In a first test, a periodic motion from left to right has been considered. Such a motion has been defined by considering the center of the end effector starting at coordinates $X_{i}=[0.05,-0.35,-0.23] \mathrm{m}$ and stopping its motion at coordinates $X_{f}=[0.35,-0.05,-0.23] \mathrm{m}$; the period of the full motion (roundtrip) requires about $2 \mathrm{~s}$ 
equally distributed in the forward motion (lasting one second) and the backward motion (lasting $1 \mathrm{~s}$ ). We have considered a failure of cable 4 occurring at $t=0.65 \mathrm{~s}$. In Figure 7 the full motion performed by the end-effector is shown by a blue line, while the instant of failure is depicted in red.

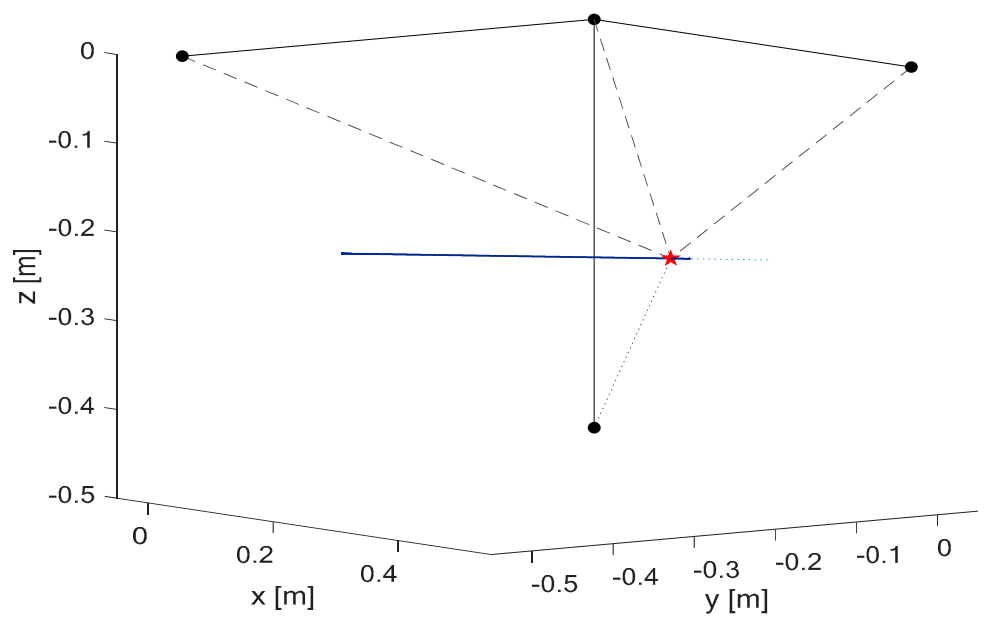

Figure 7. The computed end-effector trajectory for the proposed simulation test.

Dashed lines in Figure 7 refer to cables that are still active after the failure of another cable. The damaged or broken cable has been highlighted with a dotted line. The absolute velocity of the whole path has been plotted in Figure 8 by showing the motion before the cable failure as phase 1 (from 0 to $0.65 \mathrm{~s}$ ); the motion after cable failure as phase 2 (from 0.65 to $0.7 \mathrm{~s}$ ); the reaching of final configuration as phase 3 (from 0.7 to $1 \mathrm{~s}$ ). The tensions of the cables are depicted in Figure 9. The time axis lasts $1 \mathrm{~s}$ in order to highlight the three different phases. During the first phase the end-effector is moving along the planned trajectory; in the second phase, after the cable failure, the proposed algorithm computes the cable tensions that are needed for exerting the braking force that is needed to stop the end-effector. The last phase begins when the velocity of the end-effector is close to zero. In this phase the cable tension reaches a static equilibrium configuration in the actual position.

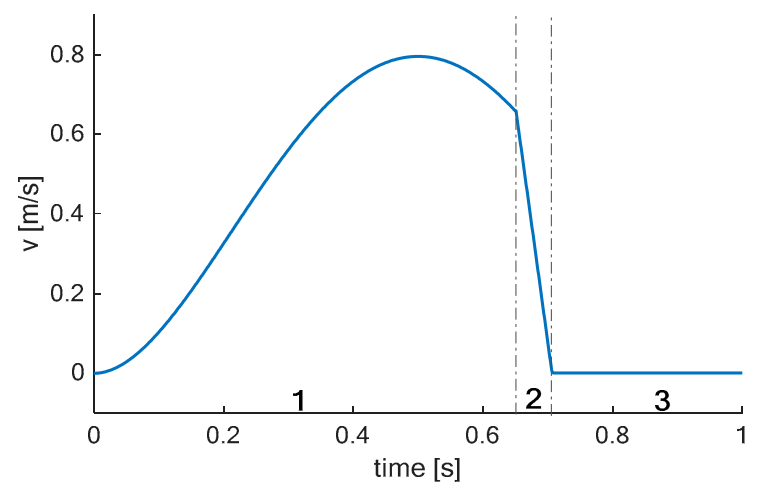

Figure 8. The end-effector velocity during the simulation (phase 1: motion before the cable failure; phase 2: motion after cable failure; phase 3: reaching of final position). 


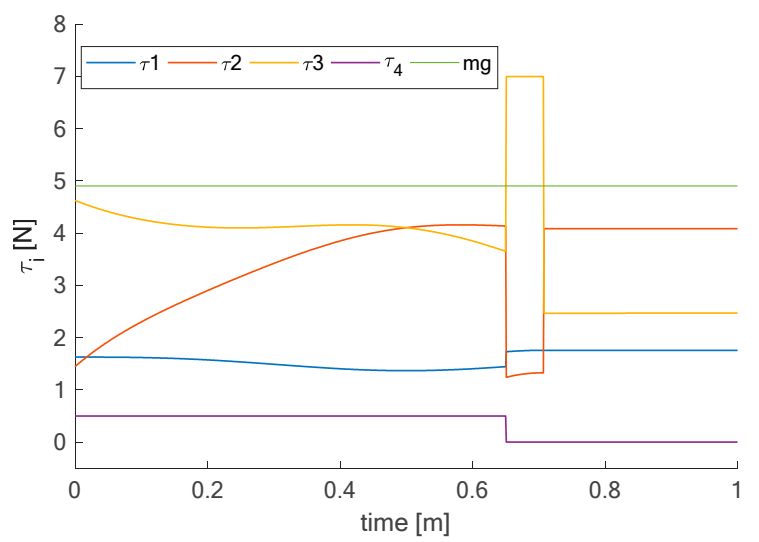

Figure 9. Cable tensions during the simulation (phase 1: motion before the cable failure; phase 2: motion after cable failure; phase 3: reaching of final position).

\section{Conclusions}

This paper addresses the safe operation of cable-driven parallel manipulators by taking into account the effect of a cable failure. Specifically, we propose a strategy to deal with one cable failure. This strategy consists of a real-time computation that, after detecting a cable failure, generates a cable tension that can achieve quick braking of the end-effector in a safe pose. A computation algorithm has been discussed and simulation results have been analyzed referring to the real case study of a LAWEX robot. This has allowed us to confirm the engineering feasibility and effectiveness of the proposed strategy when a cable failure occurs.

Author Contributions: Conceptualization, G.B., G.C. and C.P.; Investigation G.B., G.C. and C.P.; Methodology, G.B., G.C. and C.P.; G.B., G.C. and C.P. wrote the paper.

Funding: This work was supported by the Project ID 37_215, MySMIS code 103,415 “Innovative approaches regarding the rehabilitation and assistive robotics for healthy ageing" co-financed by the European Regional Development Fund through the Competitiveness Operational Program 2014-2020, Priority Axis 1, Action 1.1.4, through the financing contract 20/01.09.2016, between the Technical University of Cluj-Napoca and ANCSI as Intermediary Organism in the name and for the Ministry of European Funds.

Conflicts of Interest: The authors declare no conflict of interest.

\section{References}

1. Cone, L.L. Skycam-an aerial robotic camera system. Byte 1985, 10, 122.

2. Rosati, G.; Gallina, P.; Rossi, A.; Masiero, S. Wire-based robots for upper-limb rehabilitation. Int. J. Assist. Robot. Mechatron. 2006, 7, 3-10.

3. Boschetti, G. A picking strategy for circular conveyor tracking. J. Intell. Robot. Syst. 2016, 81, 241-255. [CrossRef]

4. Roberts, R.G.; Graham, T.; Lippitt, T. On the inverse kinematics, statics, and fault tolerance of cable-suspended robots. J. Field Robot. 1998, 15, 581-597. [CrossRef]

5. Notash, L. Failure recovery for wrench capability of wire-actuated parallel manipulators. Robotica 2012, 30, 941-950. [CrossRef]

6. Notash, L. Wrench recovery for wire-actuated parallel manipulators. Proc. Romansy 19 Robot Des. Dyn. Control 2013, 201-208. [CrossRef]

7. Ghaffar, A.; Hassan, M. Failure analysis of cable based parallel manipulators. Appl. Mech. Mater. 2014, 736, 203-210. [CrossRef]

8. Boschetti, G.; Passarini, C.; Trevisani, A. A recovery strategy for cable driven robots in case of cable failure. Int. J. Mech. Control 2017, 18, 41-48.

9. Passarini, C.; Zanotto, D.; Boschetti, G. Dynamic Trajectory Planning for Failure Recovery in Cable-Suspended Camera Systems. J. Mech. Robot. 2019. [CrossRef] 
10. Carbone, G.; Gherman, B.; Ulinici, I.; Vaida, C.; Pisla, D. Design Issues for an Inherently Safe Robotic Rehabilitation Device. In International Conference on Robotics in Alpe-Adria-Danube Region; Spinger: Dorchect, The Netherlands, 2017; pp. 967-974.

11. Carbone, G.; Arostegui Cavero, C.; Ceccarelli, M.; Altuzarra, O. A study of Feasibility for a Limb Exercising Device. Mech. Mach. Sci. 2017, 47, 11-21.

12. Boschetti, G.; Trevisani, A. Cable robot performance evaluation by wrench exertion capability. Robotics 2018, 7, 15. [CrossRef]

13. Boschetti, G.; Passarini, C.; Trevisani, A.; Zanotto, D. A Fast Algorithm for Wrench Exertion Capability Computation. In Cable-Driven Parallel Robots; Springer: Cham, Switzerland, 2018; pp. 292-303.

14. Trevisani, A. Planning of dynamically feasible trajectories for translational, planar, and underconstrained cable-driven robots. J. Syst. Sci. Complex. 2018, 26, 695-717. [CrossRef]

15. Scalera, L.; Gallina, P.; Seriani, S.; Gasparetto, A. Cable-Based Robotic Crane (CBRC): Design and Implementation of Overhead Traveling Cranes Based on Variable Radius Drums. IEEE Trans. Robot. 2018, 34, 474-485. [CrossRef]

16. Major, K.A.; Major, Z.Z.; Carbone, G.; Pisla, A.; Vaida, C.; Gherman, B.; Pisla, D.L. Ranges of Motion as Basis for robot-assisted post-stroke rehabilitation. Hum. Vet. Med. 2016, 8, 192-196.

17. Carbone, G.; Ceccarelli, M.; Rodríguez León, J.F.; Lazar, V.A.; Cafolla, D.; Vaida, C.; Pisla, D. Experimental Characterization of Assisted Human Arm Exercises. In Proceedings of the 2018 IEEE International Conference on Automation, Quality and Testing, Robotics (AQTR), Cluj-Napoca, Romania, 24-26 May 2018.

18. Rodríguez León, J.F.; Carbone, G.; Cafolla, D.; Russo, M.; Ceccarelli, M.; Castillo Castañeda, E. Experiences and Design of a Cable-Driven Assisting Device for Arm Motion. Cism Int. Cent. Mech. Sci. Courses Lect. 2019, 584, 94-101.

19. Laribi, M.A.; Carbone, G.; Zeghloul, S. Optimal design of cable driven robot for rehabilitation with prescribed workspace. Mech. Mach. Sci. 2019, 65, 273-282.

(C) 2019 by the authors. Licensee MDPI, Basel, Switzerland. This article is an open access article distributed under the terms and conditions of the Creative Commons Attribution (CC BY) license (http:// creativecommons.org/licenses/by/4.0/). 